\title{
On the Hyperinvariant Subspaces for Isometries
}

\section{Ronald GEORge Douglas}

Let $\mathscr{H}$ be a complex Hilbert space. A (bounded linear) operator $V$ on $\mathscr{H}$ is an isometry if $\|V x\|=\|x\|$ for $x$ in $\mathscr{H}$. In this note we determine the hyperinvariant subspaces for a general isometry. Recall that a subspace $\mathscr{M}$ of $\mathscr{H}$ is said to be hyperinvariant for an operator $T$ on $\mathscr{H}$ if $\mathscr{M}$ is invariant for every operator that commutes with $T$. This notion was introduced in [5] and [2], where the hyperinvariant subspaces for various classes of operators were determined. In particular, it was shown in [2] that the hyperinvariant subspaces for a unitary operator (in fact, any normal operator) are the spectral subspaces and in [5] (without proof) and [2] that the hyperinvariant subspaces for the unilateral shift $U_{+}$on $\mathrm{H}_{\mathscr{D}}$ are the subspaces $q \mathrm{H}_{\mathscr{Z}}$, where $q$ is a scalar inner function.

Before stating the characterization for general isometries we need the following facts about isometries and unitary operators. For a Hilbert space $\mathscr{D}$ we let $\mathrm{H}_{\mathscr{D}}$ denote the space of functions $f$ from the non negative integers $Z^{+}$to $\mathscr{D}$ so that $\sum_{n=0}^{\infty}\|f(n)\|^{2}<\infty$. The space $\mathrm{H}_{\mathscr{D}}$ is a Hilbert space with respect to pointwise addition and scalar multiplication and the inner product $\langle f, g\rangle=\sum_{n=0}^{\infty}(f(n), g(n))$. The unilateral shift $U_{+}$is defined on $\mathrm{H}_{\mathscr{D}}$ so that

$$
\left(U_{+} f\right)(n)=\left\{\begin{array}{cc}
0, & n=0 \\
f(n-1), & n>0
\end{array}, \quad \text { for } f \text { in } \mathrm{H}_{\mathscr{D}} .\right.
$$

The operator $U_{+}$is an isometry and its adjoint, the backward shift, satisfies $\left(U_{+}^{*} f\right)(n)=f(n+1)$ for $f$ in $\mathrm{H}_{\mathscr{D}}$. The sequence $\left\{U_{+}^{* n}\right\}$ converges strongly to 0 . The minimal unitary extension $U$ of $U_{+}$is the bilateral shift defined on $\mathrm{L}_{\mathscr{D}}$, where $\mathrm{L}_{\mathscr{D}}$ is the space of function $f$ from the integers $\mathbf{Z}$ to $\mathscr{D}$ so that $\sum_{n=-\infty}^{\infty}\|f(n)\|^{2}<\infty$ and $U$ is defined $(U f)(n)=f(n-1)$ for $f$ in $L_{\mathscr{Q}}$. It is easily verified that $U$ is unitary and if we identify $\mathrm{H}_{\mathscr{D}}$ as a subspace of $\mathrm{L}_{\mathscr{D}}$ in the obvious way, then $U_{+}=U \mid \mathrm{H}_{\mathscr{O}}$.

A result due to von Neumann [4] states that every isometry is of the form $U_{+} \oplus W$ on $\mathrm{H}_{\mathscr{T}} \oplus \mathscr{K}$, where $U_{+}$is the unilateral shift on $\mathrm{H}_{\mathscr{D}}$ and $W$ is a unitary operator on $\mathscr{K}$.

A further decomposition of the unitary part into its absolutely continuous and singular parts will be of interest. If $W$ is a unitary operator on $\mathscr{K}$ with spectral measure $E(\delta)$, then $W$ is said to be absolutely continuous [singular] if the measure $\mu(\delta)=(E(\delta) x, x)$ is absolutely continuous [singular] for each 
vector $x$ in $\mathscr{K}$. If $W$ is a unitary operator on $\mathscr{K}$, then $\mathscr{K}=\mathscr{K}_{a} \oplus \mathscr{K}_{s}$, where $\mathscr{K}_{a}$ and $\mathscr{K}_{s}$ are reducing subspaces for $W$ so that $W / \mathscr{K}_{a}$ is absolutely continuous while $W / \mathscr{K}_{s}$ is singular. The operator $W / \mathscr{K}_{a}$ is said to be the absolutely continuous part of $W$. (See [3] for details and proofs.)

Theorem. Let $V$ be an isometry on $\mathscr{H}$ and let $\mathscr{H}=\mathrm{H}_{\mathscr{D}} \oplus \mathscr{K} 1 \oplus \mathscr{K}_{2}$ be the unique decomposition of $\mathscr{H}$ into reducing subspaces for $V$ so that $U_{+}=V / \mathrm{H}_{\mathscr{D}}$ is the unilateral shift on $\mathrm{H}_{\mathscr{D}}, W_{1}=V / \mathscr{K}_{1}$ is an absolutely continuous unitary operator and $W_{2}=V / \mathscr{K}_{2}$ is a singular unitary operator. The hyperinvariant subspaces for $V$ are of the form $\mathscr{M} \oplus \mathscr{K}_{1} \oplus F \mathscr{K}_{2}$ or $(0) \oplus E \mathscr{K}_{1} \oplus F \mathscr{K}_{2}$, where $\mathscr{M}$ is hyperinvariant for $U_{+}, E$ is a spectral-projection for $W_{1}$, and $F$ is a spectral projection for $W_{2}$.

Proof. We begin by observing that the hyperinvariant subspaces for a direct sum $T_{1} \oplus T_{2} \oplus T_{3}$ are always of the form $\mathscr{M}_{1} \oplus \mathscr{M}_{2} \oplus \mathscr{M}_{3}$, where each of $\mathscr{M}_{1}, \mathscr{M}_{2}$, and $\mathscr{M}_{3}$ is hyperinvariant for the respective operator. This is because the projections $I \oplus 0 \oplus 0,0 \oplus I \oplus 0$, and $0 \oplus 0 \oplus I$ and the operators $S_{1} \oplus 0 \oplus 0$, $0 \oplus S_{2} \oplus 0$, and $0 \oplus 0 \oplus S_{3}$ commute with $T_{1} \oplus T_{2} \oplus T_{3}$ where $S_{i}$ is any operator which commutes with $T_{i}$. Thus we may assume that a hyperinvariant subspace for $V$ is of the form $\mathscr{M} \oplus E \mathscr{K}_{1} \oplus F \mathscr{K}_{2}$, where $\mathscr{M}$ is hyperinvariant for $U_{+}$and $E$ and $F$ are spectral projections for $W_{1}$ and $W_{2}$, respectively. Our task is now to decide which of these subspaces is hyperinvariant for $V$.

To this end we need to determine the commutant for $V$. The unitary extension of $V$ is $U \oplus W_{1} \oplus W_{2}$ on $\mathrm{L}_{\mathscr{D}} \oplus \mathscr{K}_{1} \oplus \mathscr{K}_{2}$, where $U$ is the bilateral shift on $\mathrm{L}_{\mathscr{g}}$. From Corollary 5.4 of [1] (cf. [6]) it follows that if $A_{+}$commutes with $V$, then $A_{+}=A / \mathscr{H}$ where $A$ is an operator on $\mathrm{L}_{\mathscr{D}} \oplus \mathscr{K}_{1} \oplus \mathscr{K}_{2}$ which commutes with $U \oplus W_{1} \oplus W_{2}$. Since a bilateral shift is an absolutely continuous unitary operator (its spectral measure is equivalent to Lebesque measure), from Lemma 4.1 of [1] it follows that the matrix for $A$ relative to the decomposition $\mathrm{L}_{\mathscr{Q}} \oplus \mathscr{K}_{1} \oplus \mathscr{K}_{2}$ is of the form

$$
\left(\begin{array}{ccc}
A_{1} & A_{2} & 0 \\
A_{3} & A_{4} & 0 \\
0 & 0 & A_{5}
\end{array}\right) .
$$

Thus it is clear that the spectral projection $F$ is independent of $\mathscr{M}$ and $E$. Thus we can confine our attention to the subspace $\mathrm{H}_{\mathscr{D}} \oplus \mathscr{K}_{1}$ and the matrix

$$
\left(\begin{array}{ll}
A_{1} & A_{2} \\
A_{3} & A_{4}
\end{array}\right) .
$$

If this matrix is to represent an operator which commutes with

$$
\left(\begin{array}{cc}
U & 0 \\
0 & W_{1}
\end{array}\right)
$$

then $A_{1}$ must commute with $U, A_{4}$ must commute with $W_{1}$, and $A_{2}$ and $A_{3}$ must satisfy $A_{2} W_{1}=U A_{2}$ and $A_{3} U=W_{1} A_{3}$. Further, if this is to be the extension of an operator which commutes with $U_{+} \oplus W_{1}$, then it must leave $\mathrm{H}_{\mathscr{D}} \oplus \mathscr{K}_{1}$ 
invariant. This implies upon computing that $A_{2} \mathscr{H}_{1}$ must be contained in $\mathrm{H}_{\mathscr{D}}$. But by Lemma 4.1 of [1] the closure of $A_{2} \mathscr{H}_{1}$ is a reducing subspace for $U$. Since the only reducing subspace for $U$ contained in $\mathrm{H}_{\mathscr{D}}$ is $(0)$ it follows that $A_{2}=0$. Lastly, we have that $\mathbf{H}_{\mathscr{D}}$ is an invariant subspace for $A_{1}$.

The preceding argument is reversible to obtain that the commutant of $U_{+} \oplus W_{1}$ consists of the restriction of operators with matrix

$\left(\begin{array}{cc}A_{1} & 0 \\ A_{3} & A_{4}\end{array}\right)$, where $A_{1} U=U A_{1}, A_{1} \mathrm{H}_{\mathscr{T}} \subset \mathrm{H}_{\mathscr{D}}, A_{3} U=W_{1} A_{3}$ and $A_{4} W_{1}=W_{1} A_{4}$.

We return now to the problem of determining which subspaces of the form $\mathscr{M} \oplus E \mathscr{K}_{1}$ are invariant under these operators. If $f \oplus g$ is in $\mathscr{M} \oplus E \mathscr{K}_{1}$, then

$$
\left(\begin{array}{cc}
A_{1} & 0 \\
A_{3} & A_{4}
\end{array}\right)\left(\begin{array}{l}
f \\
g
\end{array}\right)=\left(\begin{array}{c}
A_{1} f \\
A_{3} f+A_{4} g
\end{array}\right)
$$

so that $\mathscr{M}$ can be any hyperinvariant subspace for $U_{+}$. If $\mathscr{M}=(0)$, then $E \mathscr{K}_{1}$ can be any spectral subspace for $W_{1}$. The proof will be complete if we show for $\mathscr{M} \neq(0)$ that the set of vectors of the form $A_{3} f$, where $A_{3}$ satisfies $A_{3} U=$ $W_{1} A_{3}$ and $f$ is in $\mathscr{M}$, is dense in $\mathscr{K}_{1}$.

Choose $x$ in $\mathscr{K}_{1}$ and let $\mathscr{G}$ be the minimal reducing subspace for $W_{1}$ containing $x$. Since $W_{1}$ is absolutely continuous, there exists a Borel set $\gamma$ contained in the unit circle so that if we define $v(\delta)=m(\delta \cap \gamma)$ where $m$ is normalized Lebesgue measure, then $W_{1} / \mathscr{G}$ is unitarily equivalent to the operator $L_{z}$ defined as multiplication by $z$ on $L^{2}(v)$. Let $C$ be an isometry from $\mathscr{G}$ onto $L^{2}(v)$ so that $L_{z} C=C W_{1} / G$.

If $\mathscr{M} \neq(0)$, then $\mathscr{M} \neq U \mathscr{M}$ and if $f$ is a unit vector in $\mathscr{M} \ominus U \mathscr{M}$, then $\left\{U^{n} f\right\}_{n=-\infty}^{\infty}$ is an orthonormal subset of $\mathscr{K}_{1}$. The subspace $\mathscr{F}$ of $\mathscr{K}_{1}$ spanned by the vectors $\left\{U^{n} f\right\}_{n=-\infty}^{\infty}$ reduces $W_{1}$. For $k$ a bounded function in $L^{2}(v)$, we define an operator $D_{k}$ from $L_{\mathscr{D}}$ to $\mathscr{K}_{1}$ as follows: for $h$ orthogonal to $\mathscr{F}$ we set $D_{k} h=0$; for $h=\sum_{n=-\infty}^{\infty} \alpha_{n} U^{n} f$, we set $D_{k} h=C^{*}\left(k \sum_{n=-\infty}^{\infty} \alpha_{n} e^{\text {int }}\right)$. To show that $D_{k}$ is bounded observe that

$$
\begin{aligned}
\left\|D_{k} h\right\|^{2} & =\left\|C^{*}\left(k \sum_{n=-\infty}^{\infty} \alpha_{n} e^{\mathrm{int}}\right)\right\|^{2}=\left\|k\left(\sum_{n=-\infty}^{\infty} \alpha_{n} e^{\mathrm{int}}\right)\right\|^{2}=\frac{1}{2 \pi} \int_{\gamma}\left|k\left(\sum_{n=-\infty}^{\infty} \alpha^{n} e^{\mathrm{int}}\right)\right|^{2} d m \\
& \leqq\|k\|_{\infty}^{2} \frac{1}{2 \pi} \iint_{\gamma}\left|\sum_{n=-\infty}^{\infty} \alpha_{n} e^{\mathrm{int}}\right|^{2} d m \leqq\|k\|_{\infty}^{2} \frac{1}{2 \pi} \int_{0}^{2 \pi}\left|\sum_{n=-\infty}^{\infty} \alpha_{n} e^{\mathrm{int} t}\right|^{2} d m \\
& =\|k\|_{\infty}^{2}\left(\sum_{n=-\infty}^{\infty}\left|\alpha_{n}\right|^{2}\right)=\|k\|_{\infty}^{2}\left\|\sum_{n=-\infty}^{\infty} \alpha_{n} U^{n} f\right\|^{2}=\|k\|_{\infty}^{2}\|h\|^{2} .
\end{aligned}
$$

An easy computation shows that $D_{k} U=W_{1} D_{k}$ so that the operator on $\mathrm{H}_{\mathscr{P}} \oplus \mathscr{K}_{1}$ with matrix

$$
\left(\begin{array}{cc}
0 & 0 \\
D_{k} & 0
\end{array}\right)
$$


commutes with $U_{+} \oplus W_{1}$. Moreover

$$
\left(\begin{array}{cc}
0 & 0 \\
D_{k} & 0
\end{array}\right)\left(\begin{array}{l}
f \\
0
\end{array}\right)=\left(\begin{array}{c}
0 \\
C^{*} k
\end{array}\right)
$$

so that the set of vectors of the form $A_{3}(f \oplus 0)$, where $A_{3}$ commutes with $U_{+} \oplus W_{1}$ and $f$ is in $\mathscr{M}$, is dense in $\mathscr{G}$. Since $x$ was arbitrary, the set is dense in $\mathscr{K}_{1}$ and the proof is complete.

The preceding argument could be used to prove the "same result" for those subnormal operators having the property that every commuting map "lifts" to the minimal normal extensions. For a general subnormal the above argument shows that every hyperinvariant subspace is of this form but the possibility of further operators in the commutant obviates the argument showing that each of these subspaces is hyperinvariant,

\section{Bibliography}

1. Douglas, R. G.: On the operator equation $S^{*} X T=X$ and related topics. Acta Sci. Math. (Szeged) (to appear).

2. - , and C. M. Pearcy: On a topology for invariant subspaces. J. Functional Anal (to appear).

3. Halmos, P. R.: Introduction to Hilbert space and the theory of spectral multiplicity. New York: Chelsea 1951.

4. Neumann, J.von: Allgemeine Eigenwertheorie Hermitescher Funktionaloperatoren. Math. Ann. 102, 49-131 (1929).

5. Sz.-Nagy, B., et C. Foiaş: Analyse harmonique des opérateurs de l'espace de Hilbert. Budapest: Akademiai Kiado 1967.

6. Sz.-Nagy, B., et C. Foiaş: Dilation des commutants d'opérateurs. C. R. Acad. Sci., Paris 266, $493-495$ (1968).

Prof. R. G. Douglas

Department of Mathematics

The University of Michigan

Ann Arbor, Michigan 48104 (USA) 